\title{
Acute effects of multi-walled carbon nanotubes on primary bronchial epithelial cells from COPD patients
}

Seraina Beyeler ${ }^{1,2}$, Savvina Chortarea ${ }^{3,4}$, Barbara Rothen-Rutishauser ${ }^{3}$, Alke

Petri-Fink ${ }^{3}$, Peter Wick ${ }^{4}$, Stefan A. Tschanz ${ }^{5}$, Christophe von Garnier ${ }^{1,2,}$, Fabian Blank $^{1,2, \$, *}$

${ }^{1}$ Department of BioMedical Research, University of Bern, Bern, Switzerland

${ }^{2}$ Department of Pulmonary Medicine, University Hospital of Bern, Bern, Switzerland

${ }^{3}$ BioNanomaterials, Adolphe Merkle Institute, University of Fribourg, Fribourg, Switzerland

${ }^{4}$ Empa Materials, Science and Technology, St. Gallen, Switzerland

${ }^{5}$ Institute of Anatomy, University of Bern, Switzerland

${ }^{\S} \mathrm{Cv} G$ and FB contributed equally to this work.

Seraina Beyeler, Department of BioMedical Research, Murtenstrasse 50, 3008 Bern.

+41 3163276 34, seraina.beyeler@dbmr.unibe.ch

Savvina Chortarea, EMPA, Lerchenfeldstr. 5, CH-9014 St. Gallen.

+415876573 99, savvina.chortarea@empa.ch

Prof. Barbara Rothen-Rutishauser, Adolphe Merkle Institute, Chemin des Verdiers 4, 1700 Fribourg.

+412630095 02, barbara.rothen@unifr.ch

Prof. Alke Petri-Fink, Adolphe Merkle Institute, Chemin des Verdiers 4, 1700 Fribourg. +412630095 01. alke.fink@unifr.ch

Dr. Peter Wick, EMPA, Lerchenfeldstr. 5, CH-9014 St. Gallen.

+415876576 84, peter.wick@empa.ch

This document is the accepted manuscript version of the following article: Beyeler, S., Chortarea, S., Rothen-Rutishauser, B., Petri-Fink, A., Wick, P., Tschanz, S. A., ... Blank, F. (2018). Acute effects of multi-walled carbon nanotubes on primary bronchial epithelial cells from COPD patients.

Nanotoxicology. http://doi.org/10.1080/17435390.2018.1472310 
PD Dr. med. / Software Ing. FH Stefan A. Tschanz, Institute of Anatomy, Balzerstrasse 2, 3000 Bern.

+413163184 78, tschanz@ana.unibe.ch

Prof. Dr. med Christophe von Garnier, Department of Pulmonary Medicinie, University Hospital of Bern, Tiefenaustrasse 112, 3004 Bern.

+41313088780, christophe.vongarnier@insel.ch

* Corresponding author: PD Dr. Fabian Blank, Department of BioMedical Research, Murtenstrasse 50, 3008 Bern.

+41 3163276 34, fabian.blank@dbmr.unibe.ch 
Abstract

The risks of occupational exposure during handling of multi-walled carbon nanotubes (MWCNTs) have received limited attention to date, in particular for potentially susceptible individuals with highly prevalent chronic obstructive pulmonary disease (COPD). In this in vitro study, we simulated acute inhalation of MWCNTs employing an air-liquid interface cell exposure (ALICE) system: Primary human bronchial epithelial cells from COPD patients and healthy donors (controls), cultured at the air-liquid interface (ALI) were exposed to MWCNTs. To study acute health effects on the respiratory epithelium, two different concentrations $\left(0.16 \mu \mathrm{g} / \mathrm{cm}^{2} ; 0.34 \mu \mathrm{g} / \mathrm{cm}^{2}\right)$ of MWCNTs were aerosolized onto cell cultures followed by analysis after twenty-four hours. Following MWCNT exposure, epithelial integrity and differentiation remained intact. Electron microscopy analyses identified MWCNTs both extra- and intracellular within vesicles of mucus producing cells. In both COPD and healthy control cultures, MWCNTs neither caused increased release of lactate dehydrogenase $(\mathrm{LDH})$, nor alterations in inflammatory responses, as measured by RNA expression and protein secretion of the cytokines IL-6, IL-8, CXCL10, IL-1 $\beta$ and TGF- $\beta$ and oxidative stress markers HMOX-1 and SOD-2. No short-term alteration of epithelial cell function, as determined by ciliary beating frequency (CBF), occurred in any of the conditions tested.

In conclusion, the present study provided a reliable and realistic in vitro acute-exposure model of the respiratory tract, responsive to positive controls such as Dörentruper Quartz (DQ12) and Asbestos. Acute exposure to MWCNTs did not affect epithelial integrity, nor induce increased cell death, apoptosis or inflammatory changes.

Keywords: Exposure, Fiber Toxicology, Nanoparticles, Nanotubes, Air liquid interface cell exposure system. 


\section{Introduction}

Due to their strength and hydrophobic properties, multi-walled carbon nanotubes (MWCNTs) are promising engineered nanomaterials in industrial, recreational, and medical applications. The expansion of manufacturing volumes and applications for MWCNTs require that potential occupational health effects undergo thorough investigation and scrutiny. Furthermore, the very unique characteristics that render MWCNTs attractive compounds for novel applications may also trigger possible hazardous effects on biological systems, mainly due to the stiffness and fiber-like structure (Fadeel et al. 2007). MWCNTs as components of a solid material (e.g. recreational equipment) seem not to represent a risk to the final user, but during manufacturing or recycling processes, this nanomaterial may become airborne and potentially pose a hazard in an occupational setting, as demonstrated in different studies (Oberdörster et al. 2015; Erdely et al. 2013). The respiratory tract is considered as the main portal of entry for MWCNTs (Oberdörster 2001). The fact that MWCNTs constitute high aspect ratio nanoparticles (HARN) with asbestos-like morphology once they agglomerate to longer fibers, has fueled growing concerns related to possible asbestos-like pathogenicity (Donaldson et al. 2010) as well as cytotoxic and pro-inflammatory effects (Boyles et al. 2015). Hussain et al. (Hussain et al. 2014) reported induction of inflammasome-dependent pro-fibrotic response after MWCNT exposure. Different in vivo and in vitro studies reported oxidative stress, (pro-)inflammation, fibrosis, granulomas and even mesothelioma after MWCNT exposure (Luanpitpong et al. 2016; Albini et al. 2015). Nanomaterials, and MWCNTs in particular, may represent an additional health risk in susceptible persons with chronic respiratory disorders such as chronic obstructive pulmonary disease (COPD). COPD is a major and increasingly prevalent health problem, predicted to advance to the third leading cause of death worldwide by 2030 (WHO 2017) and characterized by an enhanced chronic inflammatory pulmonary response to noxious particles (Steiropoulos et al. 2014). Globally a considerable and increasing part of the active working population suffers from COPD and 
may be susceptible to effects of occupational airborne particulates such as MWCNTs during the manufacturing and recycling processes. The majority of publications dealing with healtheffects of industrial MWCNTs on human lung cells were performed in vitro by employing submerged cultures of cell lines with limited approach to monitor quantity of particles deposited on cells (Clift et al. 2014; Snyder-talkington et al. 2013; Thurnherr et al. 2011). Such suspension exposures have limited value to simulate inhalation of airborne particulates into the lung, followed by deposition on the lung epithelium. Consequently, an optimized MWCNT exposure model for the respiratory tract will be needed. We employed primary in vitro differentiated airway epithelial cells from healthy individuals and COPD patients. Cells cultured at the air-liquid interface were exposed to MWCNT aerosol using the air-liquid interface cell exposure system (ALICE) that enables on-line determination of the deposited particle dose. The latter enabled application of a particle dose that was comparable to ones found during occupational exposure. As a proof-of-principle, this study focused in particular on investigating short-term effects of MWCNTs on primary bronchial epithelial cells with the specific objective to monitor differentiation, integrity, functionality (ciliary beat frequency, $\mathrm{CBF}$ ) of the epithelium and assess the viability of the cell cultures. Furthermore, key biological endpoints for nanomaterial safety assessment and critical mediators involved in lung inflammatory disease such as (pro-)inflammatory (IL-6, IL-8, CXCL10, IL-1 $\beta$, TGF- $\beta$ ) and oxidative stress markers (HMOX-1, SOD-2) were characterized. To confirm responsiveness of the system, asbestos and crystalline Dörentruper quartz (DQ12), both wellknown for their pathogenic effects in vivo, were used as established positive controls for inflammatory responses (Monteiller et al. 2007; Endes et al. 2014).

To the best of our knowledge, this is the first report in the field that investigated acute effects of occupational MWCNT exposure in primary epithelial lung cell cultures from potentially susceptible individuals with COPD. 


\section{Material and Methods}

\section{Lung cell cultures}

Primary human bronchial cells from healthy and COPD donors were obtained from Epithelix (Epithelix Sàrl, Geneva, Switzerland). Cells were cultured in $700 \mu 1$ of serum free MucilAir Culture Medium (Epithelix Sàrl, Geneva, Switzerland) on trans well inserts (24 well plate) with a pore size of $0.4 \mu \mathrm{m}$ (Corning Incorporated, Corning, MA, USA) in a two-chamber system. A total of 5 independent experiments completed. All experiments were performed at first passage with cells from 6 different healthy and 4 different COPD donors.

\section{MWCNTs and respective positive controls}

Large-scale produced MWCNTs (Cheaptubes Inc., Grafton, VT) were employed. The length of the tubes are $2-16 \mu \mathrm{m}$, with an inner diameter from $2-13 \mathrm{~nm}$ and an outer diameter from 634nm (Thurnherr et al. 2011). MWCNTs dispersed in Pluronic F127 (160 ppm) (Sigma Aldrich, Switzerland) were utilized as previously described and thoroughly characterized in terms of morphology, length, diameter, metal impurities and endotoxin levels (Thurnherr et al. 2011). The stock solution of MWCNTs $(250 \mu \mathrm{g} / \mathrm{ml})$ was diluted in Pluronic F127 to the working concentration of $25 \mu \mathrm{g} / \mathrm{ml}$.

To examine potential biological effects of the dispersant, Pluronic F127 was applied independently at a concentration of 160ppm. Additionally, Crocidolite asbestos $\left(\left(\mathrm{Na}_{2}(\mathrm{Fe}, \mathrm{Mg})_{5} \mathrm{Si}_{8} \mathrm{O}_{22}(\mathrm{OH})_{2}\right)\right.$; National Research Institute for Occupational Diseases, Johannesburg, South Africa) was utilized as a positive pro-inflammatory particle control and DQ12 as a positive (pro-)inflammatory particulate aerosol control. Asbestos was dispersed in culture medium to a stock solution of $30 \mu \mathrm{g} / \mathrm{ml}$ and $7.5 \mu \mathrm{l}$ was added in $250 \mu 1$ of culture medium in the apical chamber of the cell cultures. DQ12 was nebulized three times using the ALICE system at a concentration of $0.1 \mathrm{mg} / \mathrm{ml}$, resulting in a total deposited concentration of 
$0.6 \mu \mathrm{g} / \mathrm{cm}^{2}$. This protocol was chosen because it was not possible to nebulize a higher stock solution concentration.

\section{Air-Liquid interface cell exposure system (ALICE)}

Nebulization of MWCNTs was performed using the ALICE System as described previously (Lenz et al. 2009). For each nebulization, $1 \mathrm{ml}$ of nanomaterials suspension i.e. MWCNTs, DQ12 or $1 \mathrm{ml}$ of Pluronic F127 (160ppm) with $500 \mu \mathrm{M} \mathrm{NaCl}$ (NAAPREP® physiological saline, GlaxoSmithKline, France) was added in the nebulizer. After exposure, cells remained for twenty-four hours at $37^{\circ} \mathrm{C}$ and $5 \% \mathrm{CO}_{2}$ prior to further processing.

\section{Quantification of lactate dehydrogenase $(\mathrm{LDH})$ release}

Cell death induced by MWCNTs was assessed by measuring LDH leakage into the culture medium. Twenty-four hours after exposure with MWCNTs, culture medium was collected and LDH-activity was measured using Cytotoxicity Detection Kit from Roche Applied Science (Mannheim, Germany) according to the supplier's manual. LDH was quantified photometrically by measuring at wavelength $490 \mathrm{~nm}$, with $630 \mathrm{~nm}$ as reference wavelength. Each sample was assessed in triplicate. As a positive control, cells were treated apically with $0.2 \%$ Triton $\mathrm{X} 100$ in $\mathrm{H}_{2} \mathrm{O}$ for twenty-four hours.

\section{Immunofluorescence}

Twenty-four hours after exposure to MWCNTs or asbestos, insert membranes were cut and fixed in $70 \%$ ethanol and were stained for occludin, beta tubulin, phalloidin rhodamine and DAPI as described in the supplementary data section.

\section{Confocal laser scanning microscopy (CLSM)}

Optical sections were taken using a Zeiss LSM710 with an inverted Zeiss microscope (Axio Observer.Z1; lasers: HeNe 633nm, HeNe 543nm, Ar 488nm, and a 405nm diode laser; Carl 
Zeiss AG, Feldbach, Switzerland) with a Plan-Apochromat $63 \times 1.4$ objective. Image processing was performed using IMARIS (Bitplane AG, Zurich, Switzerland). In each experimental sample the cells were scanned in 1 to 3 random locations.

\section{Enzyme linked immunosorbent assay (ELISA)}

The released pro-inflammatory cytokines IL-6, IL-8 and CXCL10 were quantified with an ELISA Kit from R\&D Systems (Minneapolis, US) according to the manufacturer's protocol. As a positive control, cells were treated for twenty-four hours with TNF- $\alpha(1 \mu \mathrm{g} / \mathrm{ml})$ for IL-6 and IL-8 and with INF- $\gamma(1 \mu \mathrm{g} / \mathrm{ml})$ for CXCL10. TNF- $\alpha$ and IFN- $\gamma$ were dispersed in culture medium and were added to the apical chamber.

\section{RNA extraction and cDNA synthesis}

Twenty-four hours after exposure, total RNA was isolated using NucleoSpin RNA kit (Macherey-Nagel, Switzerland) according to the manufacturer's protocol. Reverse transcription (incubation $1 \mathrm{~h}$ at $37^{\circ} \mathrm{C}$ ) was carried out with the Omniscript Reverse Transcription Kit (Qiagen, Switzerland) in $10 \mu 1$ volume using a master mix consisting of 10x Buffer (Qiagen, Switzerland), dNTP mix $5 \mathrm{mM}$, random primers $3 \mu \mathrm{g} / \mu \mathrm{l}$ (Invitrogen, Switzerland), omniscript RTase $4 \mathrm{U} / \mu \mathrm{l}$ (Qiagen, Switzerland) and RNase-free water (Omniscript, Switzerland).

\section{Real-time qPCR}

Quantitative real-time polymerase chain reaction (RT-qPCR) for IL-6, IL-8, CXCL10, IL-1 $\beta$ and TGF- $\beta$ and oxidative stress markers HMOX-1 and SOD-2 was performed using Fast SYBR Green master mix (Applied Biosystems, Switzerland) in a 7500 Fast Real-Time PCR System (Life Technologies, US) as described in the supplementary data section.

\section{Transmission electron microscopy (TEM)}


Characterization of MWCNT deposition: To characterize the aerosolized MWCNT deposition, TEM copper grids were exposed to nebulized CNTs in the ALICE system. TEM was performed with a Fei Technai Spirit at 120kV (Fei Technai Spirit, Oregon, USA).

Interaction of MWCNTs with primary human cell cultures: Twenty-four hours after exposure to MWCNTs, asbestos or Pluronic F127, the exposed cells on membranes were cut from the inserts and fixed for TEM analysis as described in the supplementary data section.

\section{Assessment of ciliary beating frequency (CBF)}

Twenty-four hours after exposure to MWCNTs, asbestos or Pluronic F127 the CBF of the airliquid cell cultures was measured as described in the supplementary data section.

\section{Statistics}

All data are presented as single data points with the median and for the quantification of MWCNT deposition the mean \pm standard deviation. Statistical analysis was performed with GraphPad Prism 7 (GraphPad Software Inc., La Jolla, California, USA). For the quantification of MWCNT deposition and for the cytotoxicity assay, a one-way ANOVA followed by Tukey's HSD post hoc test was performed. For cytokine response, a nonparametric one-way analysis of variance (Kruskal-Wallis) followed by Dunns post hoc test was performed. Results were considered significant if $p<0.01$. All end points were evaluated with five different repetitions $(n=5)$, except CBF measurements $(n=3)$.

\section{Results}

\section{Characterization of aerosolized nebulized particle deposition}

During exposure of cell cultures using the ALICE, TEM grids were added to empty wells to analyze and validate the deposition of aerosolized MWCNTs. Following complete 
aerosolization onto copper grids, dried droplets comprising MWCNTs were analyzed. A uniform and homogenous deposition was observed for both concentrations $(25 \mu \mathrm{g} / \mathrm{ml}$ and $250 \mu \mathrm{g} / \mathrm{ml}$ ) used (Fig. 1 A and B). Furthermore, MWCNT deposition density augmented larger agglomerates of MWCNTs were observed with higher MWCNT concentrations (Fig. 1 B).

\section{Real-time quantification and monitoring of MWCNT deposition}

Deposited mass of MWCNTs was measured using a quartz crystal microbalance (QCM; detection limit $0.09 \mu \mathrm{g} / \mathrm{cm}^{2}, 5 \mathrm{MHz}$, Standford Research Systems, GMP SA, Renens, Switzerland), integrated in the exposure chamber for real-time monitoring of deposited mass inside the cell culture well. Aerosolization in the ALICE was performed using $1 \mathrm{ml}$ of Pluronic F127 $(160 \mathrm{mg} / \mathrm{ml})$ as a negative control and $1 \mathrm{ml}$ of MWCNTs using at low $(25 \mu \mathrm{g} / \mathrm{ml})$ and high concentration $(250 \mu \mathrm{g} / \mathrm{ml})$. As shown in Fig. $1 \mathrm{C}$, the deposition values significantly increased with higher MWCNT concentration, i.e. from $0.16 \pm 0.05 \mu \mathrm{g} / \mathrm{cm}^{2}$ to $0.34 \pm 0.08 \mu \mathrm{g} / \mathrm{cm}^{2}$ $(p<0.0001)$. Deposition values of the high MWCNT concentration $(250 \mu \mathrm{g} / \mathrm{ml})$ were significantly higher $(p<0.0001)$ than Pluronic F127 and the low concentration $(p=0.0008)$ of MWCNTs $(25 \mu \mathrm{g} / \mathrm{ml})$. Aerosolization of $0.1 \mathrm{mg} / \mathrm{ml}$ DQ12 control particulates resulted in an average deposited dose of $0.20 \mu \mathrm{g} / \mathrm{cm}^{2}$ (data not shown). DQ12 was nebulized three times and the resulting deposition was $0.60 \mu \mathrm{g} / \mathrm{cm}^{2}$. Taken together, real-time monitoring of MWCNT deposition enabled aerosol exposure of cell cultures to precise concentrations of MWCNTs.

\section{Assessing cell viability following MWCNT exposure}

The effect of MWCNTs on the viability of cultured primary airway epithelial cells was assessed by measuring LDH release into the culture medium of the lower trans-well chamber twenty-four hours after exposure (Fig. 2A). Cells permeabilized with Triton X-100 served as positive control and showed significantly increased release of LDH compared to Pluronic F127 $(p<0.0001)$. The exposure to both $0.16 \mu \mathrm{g} / \mathrm{cm}^{2}$ and $0.34 \mu \mathrm{g} / \mathrm{cm}^{2}$ of MWCNTs did neither 
increase the LDH release in healthy subjects nor in COPD patients ( $p>0.9999)$ (Fig. 2A). Also, no LDH increase was seen with asbestos $(30 \mu \mathrm{g} / \mathrm{ml})$ and DQ12 $\left(0.60 \mu \mathrm{g} / \mathrm{cm}^{2}\right)$ exposure (Fig. 2A) ( $p>0.9999)$. In summary, neither MWCNTs, nor fiber or particulate controls caused excessive cell death under the culture conditions tested.

\section{Assessment of epithelial integrity by confocal laser scanning microscopy (CLSM) and transmission electron microscopy (TEM) after MWCNT exposure}

Twenty-four hours after MWCNT exposure, cells were stained for tight junctions (occludin), nuclei (DAPI), F-actin (phalloidin rhodamine) and cilia ( $\beta$-tubulin) and visualized by CLSM or were fixed in buffered $2.5 \%$ glutaraldehyde with $1.0 \%$ osmium tetroxide for TEM. A highly differentiated and consolidated epithelium characterized by the formation of tight junctions, ciliated and mucus producing cells was present within the cultures. Qualitative assessment of micrographs did not show any changes in general morphology or expression of specific proteins caused by the exposure to MWCNTs compared to cells exposed to Pluronic F127 (Fig. 2B and C). Taken together, epithelial lung cell cultures exposed to MWCNTs maintained epithelial integrity as determined by CLSM, both in COPD and healthy controls.

\section{Validation of cellular uptake of MWCNTs}

TEM micrographs of primary airway epithelial cell cultures from COPD and control subjects showed twenty-four hours after particle exposure both localized intra- and extracellular MWCNTs (Fig. 3A and B). MWCNTs were also observed within the intracellular mucus vesicles of mucus producing cells (Fig. 3A) and single MWCNTs were also identified on the cell surface (Fig. 3B). Hence, qualitative TEM analysis confirmed that MWCNTs were primarily localized intracellular within mucus-containing vesicles.

\section{Acute cytokine responses to MWCNT exposure}


To study inflammatory responses of MWCNT-exposed air-liquid cell-cultures, culture media was collected twenty-four hours after exposure and analyzed by ELISA. Protein secretion of the pro-inflammatory cytokines, IL-6, IL-8, and CXCL10 was measured. As a positive control, asbestos $(30 \mu \mathrm{g} / \mathrm{ml})$ and TNF- $\alpha(1 \mu \mathrm{g} / \mathrm{ml})$ were used for IL-6 and IL-8, while IFN- $\gamma$ $(1 \mu \mathrm{g} / \mathrm{ml})$ was apically added as a positive control for CXCL10 and DQ12 $\left(0.60 \mu \mathrm{g} / \mathrm{cm}^{2}\right)$ was nebulized at the air-liquid surface with the ALICE system. Exposure to MWCNTs did neither alter the cytokine profile, as measured by the secretion of IL-6 ( $p>0.9999)$, IL-8 $(p>0.9999)$ nor CXCL10 $(p>0.9999)$ in both COPD and healthy control cell culture supernatant, (SI Fig.1). Also, control cell cultures with Pluronic F127 $(p>0.9999)$ and asbestos $(p>0.9999)$ did not generate a detectable alteration in cytokine patterns (SI Fig.1). In contrast positive controls, i.e. cultures treated with TNF- $\alpha$ significantly increased the release of IL-8, $(p=0.0009, p<0.0001)$ but not of IL-6 ( $p>3849, p=0.1787)$ (SI Fig.1 left and middle panels), whereas IFN- $\gamma$ significantly increased CXCL10 secretion $(p=0.0305, p=0.0004$ ) (SI Fig.1 right panel).

The relative mRNA expression of pro-inflammatory markers IL-6, IL-8, CXCL10, TGF- $\beta$ and IL-1 $\beta$ (Fig. 4A) and oxidative stress markers HMOX-1 and SOD-2 were assessed (Fig. 4B). Both MWCNT concentrations failed to induce a significant expression on any of the cytokines or oxidative stress markers, both in healthy ( $p>0.9999)$ and COPD ( $>00.9999)$ cells, as compared to the negative control (Pluronic F127-treated cultures) (Fig. 4A and B). The positive DQ12 control significantly increased expression of IL- $1 \beta$ in healthy $(p=0.0014)$ and COPD cells $(p<0.0001)$, TGF- $\beta$ in healthy $(p<0.0001)$ and COPD cells $(p<0.0001)$, IL-6 in healthy cells $(p=0.0004)$ (Fig. 4A), and SOD-2 in healthy $(p<0.0041)$ and COPD cells $(\mathrm{p}<0.0002)$ (Fig. 4B). However, DQ12 significantly upregulated IL-6 and CXCL10 only in COPD cells $(p=0.0004, p=0.00386)$, but not in healthy controls $(p=0.3077, p>0.9999)$ (Fig. 4A). For additional positive controls, IFN- $\gamma$ induced a significantly increase of CXCL10 expression in healthy $(\mathrm{p}=0.0044)$ and COPD cells $(\mathrm{p}=0.012)$. TNF- $\alpha$ induced a significantly 
increase of IL-8 expression in healthy $(p=0.0403)$ and COPD cells $(p<0.0001)$ (Fig. 4A). An asbestos control was employed throughout and did not induce significant cytokine changes, but increased expression of the oxidative stress marker HMOX-1, both in healthy $(p=0.0062)$ and COPD cells $(\mathrm{p}=0.0017)$ (Fig. 4B). In summary, none of the MWCNT concentrations utilized induced detectable short-term cytokine profiles alterations in the epithelial cell cultures when compared to multiple positive and negative controls employed.

\section{CBF after exposure to MWCNTs}

$\mathrm{CBF}$ in air-liquid cell cultures was measured twenty-four hours post-incubation time following exposure to Pluronic F127, asbestos and MWCNTs. The measurements were performed either in the center or in the periphery of the insert (Fig. 5A and B respectively). In general, CBF ranged between 5 to $10 \mathrm{~Hz}$. In the central part of the cell culture, exposure to asbestos and different MWCNT concentrations did not alter CBF when compared to the negative Pluronic 127 control (Healthy controls: Asbestos $(p>0.9999)$, low $(p>0.9999)$ and high $(p>0.9999)$ MWCNT concentration; COPD cell cultures: Asbestos $(p>0.9999)$, low $(p>0.9999)$ and high $(p>0.9999)$ MWCNTs concentrations) (Fig. 5A). No significant changes were detected upon CBF measurement in the periphery of the cell cultures (Healthy controls: Asbestos $(p>0.9999)$, low $(p>0.9999)$ and high $(p>0.9999)$ MWCNT concentration; COPD cells: Asbestos $(p>0.9999)$, low $(p>0.9999)$, and high $(p=0.2033)$ MWCNT concentration) (Fig. 5B). Interestingly, for all subjects, CBF was higher (not significant) in the periphery of the culture well (Fig. 5B) than in the central part (Fig. 5A). In conclusion, acute exposure to MWCNTs did not affect ciliary beating frequency neither in healthy nor COPD cultures.

\section{Discussion}

Although, an increasing number of studies from the recent past have documented enhanced risk for adverse health effects (Donaldson et al. 2006; Oberdörster et al. 2005; Oberdörster et 
al. 2016), there currently is insufficient data available to judge the effects of airborne MWCNTs in potentially susceptible risk groups suffering from chronic lung disorders, such as highly prevalent COPD that affects $400^{\prime} 000$ individuals in Switzerland (Lungenliga Schweiz, 2018).

With the present study, we aimed to investigate in vitro whether cells derived from individuals with COPD displayed an increased susceptibility upon MWCNT exposure when compared to cells from healthy donors. In particular, we focused on short-term (acute) effects, occurring within twenty-four hours since long-term exposure in in vitro models is limited by an increased cell mortality and artefacts. We employed primary airway epithelial cells cultured in vitro at the ALI and utilized the ALICE system to nebulize an accurately defined and characterized liquid suspension of MWCNTs on cell cultures that guarantees, a uniform deposition, as described in previous studies (Chortarea et al. 2015; Chortarea et al. 2017; Thurnherr et al. 2009; Lenz et al. 2009). The advantage of primary airway epithelial cells grown at the ALI employed in the current study lies in their ability to completely differentiate into a tight, high prismatic epithelium consisting of ciliated and mucus producing cells, a level of differentiation that is impossible to obtain with cell lines and/or with entirely submerged epithelia. In addition, this system allows a comparison of healthy cells with cells derived from COPD patients.

The most common current approaches for in vitro exposure with nanoparticles in inhalation toxicology research are the adding of suspended particles into submersed cultures, liquid droplet aerosol deposition, and dry aerosol exposure. The ALICE System provides the advantages to nebulize a suspension of nanoparticles at the air-liquid interface of cultured cells and has already been shown to be applicable for a variety of nanomaterials (Lenz et al. 2009; Chortarea et al. 2015; Endes et al. 2014). Utilizing this approach, we aimed to perform reproducible, spatial homogeneous and dose-dependent deposition on the cell surface that was controlled online with an integrated quartz crystal microbalance. For future studies it may be 
of interest to test dry powder aerosolization systems that circumvent the preparation of a dispersion as described (Polk et al. 2016; Ji et al. 2017).

In the present study, the effect of two concentrations of MWCNTs on epithelial cells was studied. The MWCNT type employed in our model is one of the most commonly produced MWCNTs that have previously been shown to induce cytotoxicity, pro-inflammatory responses and genotoxicity when applied in suspension (Johnston et al. 2010). The deposited doses for MWCNTs in the current study were $0.16 \mu \mathrm{g} / \mathrm{cm}^{2}$ and $0.34 \mu \mathrm{g} / \mathrm{cm}^{2}$ of MWCNTs, both selected in accordance to the permissible exposure limit of $1 \mu \mathrm{g} / \mathrm{m}^{3}$ suggested by the NIOSH (National Institute for Occupational Safety and Health) (NIOSH 2013). Gangwal et al. have designed and performed a study of the alveolar mass retention of a full working lifetime exposure to $1 \mu \mathrm{g} / \mathrm{cm}^{3}$ aerosol concentration of CNT in the range of 12.4 to $46.5 \mu \mathrm{g} / \mathrm{cm}^{2}$ (Gangwal et al. 2011). Therefore, the doses of deposited MWCNT utilized in our study reflect human exposure over several weeks and represent realistic concentrations that occur during manufacturing processes (Gangwal et al. 2011). One important limitation of our study lies with the fact that the cells were exposed to the total concentration only for a defined duration of twenty-four hours. The current approach, however, allowed us to solely focus on acute health effects MWCNTs may induce.

Following a thorough characterization of distribution pattern and quantity of the in vitro particle deposition, the cytotoxic capacity of MWCNTs was assessed in vitro by measuring the LDH release while the cell morphology was analyzed by CLSM and TEM following MWCNT exposure. In our cell culture model, none of the applied MWCNT concentrations induced measurable cytotoxicity. In contrast to the triton X 100 positive control, routinely integrated positive controls with asbestos (fiber control) and DQ12 (particulate control) did not lead to an increased LDH release, but confirmed the responsiveness of the cell culture. Moreover, the structure of the epithelial cell layer was maintained after twenty-four hours and we observed no alterations in the cell nuclei, tight junctions or ciliary cells. These results are 
in line with recent findings, where the effects of the same type of MWCNTs in a 3D human in vitro model were analyzed (Chortarea et al., 2015).

The visualization of the interactions between MWCNTs and cells by CLSM or TEM is important in order to accurately assess the direct impact of MWCNTs on the intra- and extracellular compartment. In our study, cell morphology as visualized by CLSM showed no structural impairment, yet analysis of cell cultures by TEM revealed interactions between MWCNTs and epithelial cells, detecting intracellular MWCNTs within mucus vesicles of mucus producing cells, and extracellular MWCNTs in the ciliary region. Chortarea et al. observed that MWCNTs utilized at similar concentrations and administered to A549 cells by ALICE were detected as single tubes inside cellular vesicles (Chortarea et al. 2015). Comparable findings were shown in primary human bronchial epithelial cell cultures from healthy and asthmatic donors where MWCNTs were found within vesicular structures (Chortarea et al. 2017). Snyder et al. detected MWCNT uptake in bronchoalveolar epithelial cells after twenty-four hours into vesicular structures, but also free in the cytoplasm (Snyder et al. 2017), which contrast with our findings. In the present study, intracellular MWCNTs detected by TEM were found to be very rare events. Therefore, we cannot exclude that a fraction of MWCNTs was localized in other sub-cellular compartments, such as early and late endosomal structures.

To test the biological sensitivity and reliability of our system in terms of its responsiveness for pro-inflammatory cytokines, viability and oxidative stress, we utilized DQ12 and asbestos as two different positive controls that generally induced expression of pro-inflammatory cytokines as reported before (Monteiller et al. 2007; Endes et al. 2014). DQ12 was employed as a positive particulate control nebulized with the ALICE system, whereas the positive fiber control asbestos could only be added in a submerged manner to the cell cultures, because it blocked the ALICE system. DQ12 exposure upregulated gene expression for IL-6 in COPD cells and TGF- $\beta$, IL-1 $\beta$ and HMOX-1 in both healthy and COPD cell cultures. SOD-2 was 
significantly upregulated in asbestos-exposed healthy and COPD cells after twenty-four hours. Expression of the IL-8 and CXCL10 genes were significantly increased in DQ12exposed COPD cells. In DQ12-exposed cell cultures, no significantly changes were seen for IL-6 $(p=0.1757)$ and CXCL10 $(p=0.5403)$ expression in healthy control cells, and for IL-8 both in healthy control and COPD cells $(p=0.3077$ and $p=0.0629$ respectively). Though the differences did not reach significances, there was an increased trend for upregulation in cytokine expression in DQ12-exposed cells. With the current findings, we showed that DQ12 upregulated expression for a range of inflammatory and oxidative stress genes in healthy and COPD cells, thereby confirming the biological responsiveness of our in vitro system. Limited effects seen in asbestos-exposed cell cultures may be due to short-term term nature of the exposure scenario utilized. Recently non-significant effects in proliferation of asbestosexposed human primary small airway epithelial cells after 7 days of treatment were reported, suggesting that asbestos-related effects operate over longer latencies (Stueckle et al. 2017).

Since MWCNTs may induce oxidative stress in human lung epithelial cells in vitro (Snydertalkington et al. 2018), we measured expression of HMOX-1 and SOD-2 that have recently been reported to play a crucial role in the development of COPD (Zhou et al. 2017). Following MWCNT exposure in healthy and COPD cell cultures, we observed no increased expression of any of the two markers studied. Also, we measured the expression of different pro-inflammatory markers, which are well known as important biological endpoints in nanomaterial safety assessment and are further critical mediators in lung inflammatory disease, such as COPD. IL-6 is one of the most important marker for acute and chronic inflammation, and plays an important role in systemic inflammation and in the pathogenesis of COPD (Wei et al. 2015). Another key marker for acute inflammation and a chemoattractant for neutrophils is IL-8 (Zhang et al. 2011). This cytokine regulates migration of neutrophils and mononuclear cells from the bronchial wall to the lumen in COPD (Di Stefano et al. 2004)(Di Stefano et al. 2004). CXCL10 is associated with airway inflammation and plays a 
crucial role as chemoattractant for the activation of NK cells and T-lymphocytes and is related to COPD exacerbation (Quint et al. 2010). TGF- $\beta$ is a fibrinogenic growth factor and multifunctional cytokine associated with differentiation, apoptosis, survival and proliferation (Königshoff et al. 2009). TGF- $\beta$ also leads to an increased deposition of extracellular matrix in the airways and an increased submucosal collagen expression (Mak et al. 2009). IL-1 $\beta$ is one of the most important and major involved cytokine in the initiation as well as the retention of an inflammation. The IL-1 $\beta$ production is enhanced in stable COPD patients, but is further increasing during exacerbation of the illness. Alveolar macrophages as well as mononuclear cells from COPD patients produce more IL-1 $\beta$ compare to healthy subjects (Lappalainen et al. 2005). We did not detect any specific changes in pro-inflammatory cytokine profiles following MWCNT exposure, neither in cells from healthy individuals, nor in those obtained from COPD patients. We cannot exclude that reduced concentrations in cytokines in healthy and COPD cells may result from short-term exposure, but this approach was deliberately employed to investigate short-term in vitro effects of MWCNTs on respiratory tract epithelial cells that are prone to increased inflammatory responses in COPD. The experimental setup for this study was based on previously published data (Gasser et al. 2012; Chortarea et al. 2017; Chortarea et al. 2015). We observed a degree of variability in pro-inflammatory cytokine expression that is likely related to the fact that primary cell cultures were derived from different donors with individual biological variation. Such donorspecific variation may be advantageous to study nanosafety and perform toxicological screening, as it reflects the genetic heterogeneity in the human population.

In the present study, we aimed to analyze the effects of MWCNTs on mucociliary motility by performing an analysis of the $\mathrm{CBF}$. Our results in measurement of $\mathrm{CBF}$ showed that the location of cells within the culture (center vs. periphery) had a higher impact on CBF than the exposure with different quantities of MWCNTs or the health status of the cells. A recent study showed that the ciliary beating frequency of human bronchial epithelial cells cultured at the 


\begin{abstract}
ALI was not affected by cellular treatment with MWCNTs after the differentiation, but if treated before total maturation, the ciliary beating frequency was reduced (Snyder et al. 2017). Such distinct changes in frequencies render the interpretation of the readouts a challenging and complex issue that requires further investigations. Beside different competent twodimensional (2D) and three-dimensional (3D) in vitro models such as ciliated human nasal epithelium or EpiAirway measuring of live $\mathrm{CBF}$ in vivo would be an appropriate alternative for in vitrolex vivo experiments in order to assess effects of CNTs in biological systems as presented by previous studies (Zavala et al. 2016; Di Benedetto et al. 1991; Navarrette et al. 2012).
\end{abstract}

\title{
Conclusion
}

Acute exposure of primary epithelial cells derived from COPD and healthy controls to occupationally relevant MWCNT doses did not alter cellular viability, epithelial integrity, inflammatory cytokine profiles, or oxidative stress markers when compared to the positive particulate control DQ12. Though this study did not detect epithelial alterations following MWCNT exposure, a comparison to extant in vivo data can be conducted to clarify effects of airborne MWCNTs on the respiratory tract (Van Berlo et al. 2014; Ma-hock et al. 2009; Vietti et al. 2013). In addition, long-term studies with repetitive MWCNT exposure will be required to assess possible occupational hazards of these materials at the working place.

\section{Acknowledgements}

The authors would like to thank the Microscopy Imaging Center (MIC) of the University of Bern for the electron microscopy sample preparation and for the performance of microscopy with devices supported by the MIC. 


\section{Declaration of interest statement}

No potential conflict of interest was reported by the authors. The authors alone are responsible for the content and writing of the paper.

This study was supported by Schweizerische Unfallversicherungsanstalt (SUVA), Bern, Switzerland, Lungenliga Schweiz, Mobiliar Jubiläumsstiftung and by a grant of the Swiss National Science Foundation (\# 310030_159847 / 1). 


\section{References}

Albini, A. et al., 2015. Environmental impact of multi-wall carbon nanotubes in a novel model of exposure: Systemic distribution, macrophage accumulation, and amyloid deposition. International Journal of Nanomedicine, 10, pp.6133-6145.

Di Benedetto, G. et al., 1991. Calcium regulation of ciliary beat frequency in human respiratory epithelium in vitro. Journal of physiology, pp.103-113.

Van Berlo, D. et al., 2014. Apoptotic, inflammatory, and fibrogenic effects of two different types of multi $\square$ walled carbon nanotubes in mouse lung. Arch Toxicol, pp.1725-1737.

Boyles, M.S.P. et al., 2015. Toxicology in Vitro Multi-walled carbon nanotube induced frustrated phagocytosis, cytotoxicity and pro-inflammatory conditions in macrophages are length dependent and greater than that of asbestos. TOXICOLOGY IN VITRO, 29(7), pp.1513-1528. Available at: http://dx.doi.org/10.1016/j.tiv.2015.06.012.

Chortarea, S. et al., 2017. Human Asthmatic Bronchial Cells Are More Susceptible to Subchronic Repeated Exposures of Aerosolized Carbon Nanotubes At Occupationally Relevant Doses Than Healthy Cells. ACS Nano, p.acsnano.7b01992. Available at: http://www.ncbi.nlm.nih.gov/pubmed/28505409\%0Ahttp://pubs.acs.org/doi/abs/10.1021 /acsnano.7b01992.

Chortarea, S. et al., 2015. Repeated exposure to carbon nanotube-based aerosols does not affect the functional properties of a 3D human epithelial airway model. Nanotoxicology, 5390(August 2016), pp.1-11.

Clift, M.J.D. et al., 2014. A Comparative Study of Different In Vitro Lung Cell Culture Systems to Assess the Most Beneficial Tool for Screening the Potential Adverse Effects of Carbon Nanotubes. Toxicological sciences : an official journal of the Society of 
Toxicology, 137(1), pp.55-64.

Donaldson, K. et al., 2010. Asbestos, carbon nanotubes and the pleural mesothelium: a review of the hypothesis regarding the role of long fibre retention in the parietal pleura, inflammation and mesothelioma. Particle and fibre toxicology, 7, p.5.

Donaldson, K. et al., 2006. Carbon nanotubes: a review of their properties in relation to pulmonary toxicology and workplace safety. Toxicological sciences : an official journal of the Society of Toxicology, 92(1), pp.5-22. Available at: http://www.ncbi.nlm.nih.gov/pubmed/16484287 [Accessed December 23, 2013].

Endes, C. et al., 2014. An in vitro testing strategy towards mimicking the inhalation of high aspect ratio nanoparticles. Available at: http://www.particleandfibretoxicology.com/content/11/1/40.

Erdely, A. et al., 2013. Carbon nanotube dosimetry : from workplace exposure assessment to inhalation toxicology., pp.1-14.

Fadeel, B. et al., 2007. There's plenty of room at the forum: Potential risks and safety assessment of engineered nanomaterials. Nanotoxicology, 1, pp.73-84. Available at: http://www.informaworld.com/openurl?genre=article \&doi=10.1080/1743539070156557 8\&magic $=$ crossref $\%$ 7C $\%$ 7CD404A21C5BB053405B1A640AFFD44AE3.

Gangwal, S. et al., 2011. Informing Selection of Nanomaterial Concentrations for ToxCast in Vitro Testing Based on Occupational Exposure Potential. , 119(11), pp.1539-1546.

Gasser, M. et al., 2012. Pulmonary surfactant coating of multi-walled carbon nanotubes (MWCNTs) influences their oxidative and pro-inflammatory potential in vitro. Particle and fibre toxicology, 9, p.17. Available at: http://www.pubmedcentral.nih.gov/articlerender.fcgi?artid=3496593\&tool=pmcentrez\&r 


$$
\text { endertype }=\text { abstract. }
$$

Ji, J. et al., 2017. Development of Combining of Human Bronchial Mucosa Models with Xpose ALI ${ }^{\circledR}$ for Exposure of Air Pollution Nanoparticles. PLoS ONE, pp.1-17.

Johnston, H.J. et al., 2010. A critical review of the biological mechanisms underlying the in vivo and in vitro toxicity of carbon nanotubes: The contribution of physico-chemical characteristics. Nanotoxicology, 4, pp.207-246.

Königshoff, M., Kneidinger, N. \& Eickelberg, O., 2009. TGF- $\beta$ signalling in COPD : deciphering genetic and cellular susceptibilities for future therapeutic regimens. Swiss Med Wkly., 9, pp.554-563.

Lappalainen, U. et al., 2005. Interleukin-1 는 Causes Pulmonary Inflammation, Emphysema , and Airway Remodeling in the Adult Murine Lung. Am J Respir Cell Mol Biol, 2.

Lenz, A.G. et al., 2009. A dose-controlled system for air-liquid interface cell exposure and application to zinc oxide nanoparticles. Particle and fibre toxicology, 6, p.32. Available at: http://www.pubmedcentral.nih.gov/articlerender.fcgi?artid=2804607\&tool=pmcentrez\&r endertype $=$ abstract [Accessed December 23, 2013].

Luanpitpong, S., Wang, L. \& Rojanasakul, Y., 2016. The effects of carbon nanotubes on lung and dermal cellular behaviors. Nanomedicin (Lond), 16(3), pp.338-348.

Lungenliga Schweiz, 2018. COPD. Available at: https://www.lungenliga.ch/de/krankheitenihre-folgen/copd.html.

Ma-hock, L. et al., 2009. Inhalation Toxicity of Multiwall Carbon Nanotubes in Rats Exposed for 3 Months. Toxicological Sciences, 112(April), pp.468-481. 
Mak, J.C.W. et al., 2009. Elevated plasma TGF- b 1 levels in patients with chronic obstructive pulmonary disease. Respiratory Medicine, 103(7), pp.1083-1089. Available at: http://dx.doi.org/10.1016/j.rmed.2009.01.005.

Monteiller, C. et al., 2007. The pro-inflammatory effects of low-toxicity low-solubility particles, nanoparticles and fine particles, on epithelial cells in vitro: the role of surface area. Occup Environ Med, 64, pp.609-615.

Navarrette, C.R. et al., 2012. Particulate Matter in Cigarette Smoke Increases Ciliary Axoneme Beating Through Mechanical Stimulation. Journal of Aerosol Medicine and Pulmonary Drug Delivery, 25, pp.159-168.

NIOSH, 2013. NIOSH: Current intelligence bulletin 65: Occupational Exposure to Carbon Nanotubes and Nanofibers.

Oberdörster, G. et al., 2015. Inhalation Exposure to Carbon Nanotubes ( CNT ) and Carbon Nanofibers ( CNF ): Methodology and Dosimetry. Journal of Toxicology and Environmental Health, Part B, 18(3-4), pp.121-212. Available at: http://dx.doi.org/10.1080/10937404.2015.1051611.

Oberdörster, G. et al., 2016. Inhalation exposure to carbon nanotubes (CNT) and carbon nanofibers (CNF): Methodology and Dosimetry. Journal of Toxicology and Environmental Health, 1848, pp.3047-3054.

Oberdörster, G., 2001. Pulmonary effects of inhaled ultrafine particles. International archives of occupational and environmental health, 74, pp.1-8.

Oberdörster, G., Oberdörster, E. \& Oberdörster, J., 2005. Nanotoxicology: an emerging discipline evolving from studies of ultrafine particles. Environmental health perspectives, 113, pp.823-839. 
Polk, W.W. et al., 2016. Aerosol generation and characterization of multi-walled carbon nanotubes exposed to cells cultured at the air-liquid interface. Particle and Fibre Toxicology, pp.1-12. Available at: http://dx.doi.org/10.1186/s12989-016-0131-y.

Quint, J.K., Donaldson, G.C. \& Goldring, J.J.P., 2010. Serum IP-10 as a Biomarker of Human Rhinovirus Infection at Exacerbation of COPD. Chest.

Snyder-talkington, B.N. et al., 2013. Multi-walled carbon nanotubes induce human microvascular endothelial cellular effects in an alveolar-capillary co-culture with small airway epithelial cells. Particle and Fibre Toxicology, 10(1), p.1.

Snyder-talkington, B.N. et al., 2018. Systematic Analysis of Multiwalled Carbon NanotubeInduced Cellular Signaling and Gene Expression in Human Small Airway Epithelial Cells. Toxicological Sciences, 133(January), pp.79-89.

Snyder, R.J. et al., 2017. Impaired Ciliogenesis in differentiating human bronchial epithelia exposed to non-Cytotoxic doses of multi-walled carbon Nanotubes. Particle and fibre toxicology, pp.1-14.

Di Stefano, A., Capelli, A. \& Donner, C.F., 2004. Role of Interleukin-8 in the Pathogenesis and Treatment of COPD. chest journal.

Steiropoulos, P. et al., 2014. New developments in the management of COPD: clinical utility of indacaterol $75 \mu \mathrm{g}$. International journal of chronic obstructive pulmonary disease, 9 , pp.1-7. Available at: http://www.pubmedcentral.nih.gov/articlerender.fcgi?artid=3862584\&tool=pmcentrez\&r endertype $=$ abstract.

Stueckle, T.A. et al., 2017. Effect of surface functionalizations of multi-walled carbon nanotubes on neoplastic transformation potential in primary human lung epithelial cells. 
Nanotoxicology, 0(0), pp.1-37. Available at:

https://www.tandfonline.com/doi/full/10.1080/17435390.2017.1332253.

Thurnherr, T. et al., 2011. A comparison of acute and long-term effects of industrial multiwalled carbon nanotubes on human lung and immune cells in vitro. Toxicology Letters, 200(3), pp.176-186.

Thurnherr, T. et al., 2009. Comprehensive evaluation of in vitro toxicity of three large-scale produced carbon nanotubes on human Jurkat T cells and a comparison to crocidolite asbestos. Nanotoxicology, 3(4), pp.319-338. Available at:

http://informahealthcare.com/doi/abs/10.3109/17435390903276958 [Accessed December 23, 2013].

Vietti, G. et al., 2013. Towards predicting the lung fibrogenic activity of nanomaterials : experimental validation of an in vitro fibroblast proliferation assay. Particle and fibre toxicology, pp.1-14.

Wei, J. et al., 2015. Association between serum interleukin-6 concentrations and chronic obstructive pulmonary disease : a systematic review and meta-analysis. Peer J.

WHO, 2017. World Health Organization. Available at: http://www.who.int/respiratory/copd/en/.

Zavala, J. et al., 2016. Assessment of Biological Responses of EpiAirway ${ }^{\text {TM }}$ 3-D Cell Constructs vs. A549 Cells for Determining Toxicity of Ambient Air Pollution. Inhal Toxicol, 28(6), pp.251-259.

Zhang, X. et al., 2011. Cytokine Increased interleukin ( IL ) -8 and decreased IL-17 production in chronic obstructive pulmonary disease ( COPD ) provoked by cigarette smoke. Cytokine, 56(3), pp.717-725. Available at: 
http://dx.doi.org/10.1016/j.cyto.2011.09.010.

Zhou, H. et al., 2017. Genetic polymorphism of heme oxygenase 1 promoter in the occurrence and severity of chronic obstructive pulmonary disease : a meta-analysis Characteristics of included studies. , 21(5), pp.894-903. 


\section{Legends}

Figure 1: Aerosolization of MWCNTs: A and B: TEM images of MWCNTs deposited in the ALICE. Nebulization of $1 \mathrm{ml}$ of MWCNT suspension was performed with two concentrations that resulted in a uniform deposition pattern. A: $25 \mu \mathrm{g} / \mathrm{ml}$ revealed a uniform disposition pattern with few agglomerates. B: $250 \mu \mathrm{g} / \mathrm{ml}$ resulted in a denser coverage and larger agglomerates. A representative set of micrographs from at least 3 independent experiments is shown. C: Quantification of MWCNT deposition using the QCM. Deposited particle mass of nebulized Pluronic (Plu) $\left(0.0995 \mu \mathrm{g} / \mathrm{cm}^{2}\right)$ (negative control), low concentration of MWCNTs (LC) $\left(0.16 \mu \mathrm{g} / \mathrm{cm}^{2}\right)$ and high concentration of MWCNTs (HC) $\left(0.34 \mu \mathrm{g} / \mathrm{cm}^{2}\right)$ were measured. Experiments were performed in triplicates. Bars show mean \pm SD of at least 3 independent experiments. Statistical significance was determined by one-way ANOVA followed by Tukey's HSD post hoc test values were considered significantly different compared to the control or compared to the low concentration with $\mathrm{p}<0.001(* * *)$ and $\mathrm{p}<0.0001(* * * *)$.

Figure 2: Cytotoxicity and morphology in airway epithelial cells exposed to MWCNTs. A: Cytotoxicity measured in primary epithelial cultures following MWCNT exposure. Extracellular LDH release was measured twenty-four hours after the exposure to low $\left(0.16 \mu \mathrm{g} / \mathrm{cm}^{2}\right)$ and high $\left(0.34 \mu \mathrm{g} / \mathrm{cm}^{2}\right)$ concentration of MWCNTs. Triton X-100 treated cells (twenty-four hours) served as positive control. LDH release was also measured for cells exposed to aerosolized DQ12 $\left(0.60 \mu \mathrm{g} / \mathrm{cm}^{2}\right)$ and treated with asbestos $(30 \mu \mathrm{g} / \mathrm{ml})$. Graph show single data points and mean from samples of individual patients. Triton X-100 (Triton), Pluronic (Plu), asbestos (Asb), DQ12 (DQ12), low concentration (LC), high concentration (HC). Statistical significance was determined by one-way ANOVA followed by Tukey's HSD post hoc test values were considered significantly different compared to the negative control Pluronic F127 with $\mathrm{p}<0.0001(* * * *)$.

B: Morphology of completely differentiated airway epithelial cells of healthy and COPD 
patients visualized by CLSM. Pluronic (Plu), asbestos (Asb), $0.16 \mu \mathrm{g} / \mathrm{cm}^{2}$ MWCNTs (LC) and $0.34 \mu \mathrm{g} / \mathrm{cm}^{2}$ MWCNTs (HC) shows reconstructions of consecutive optical sections twenty-four hours after exposure to Pluronic or MWCNTs. F-actin: red, cilia: green, occludin: white, DAPI: blue. The upper picture shows $\mathrm{x} / \mathrm{y}$-projection, the lower $\mathrm{x} / \mathrm{z}$-projection and inserts show occludin staining. One representative culture, out of at least 5 experiments, is shown from a healthy subject and a COPD patient. C: Ultrastructural morphology of airway epithelial cells after exposure to MWCNTs visualized with TEM. TEM images from primary human bronchial epithelial cells from healthy (upper row) and COPD (lower row) subjects. Micrographs show cultures exposed with Pluronic (Plu), asbestos (Asb), $0.16 \mu \mathrm{g} / \mathrm{cm}^{2}$ MWCNTs (LC) and $0.34 \mu \mathrm{g} / \mathrm{cm}^{2}$ MWCNTs (HC) for $24 \mathrm{~h}$. One representative culture, out of at least 5 experiments was chosen. Scale bars: $5 \mu \mathrm{m}$

Figure 3: A,B and A',B': Uptake of MWCNTs in primary human bronchial epithelial cells from healthy (upper row) and COPD individuals (lower row) twenty-four hours after exposure as visualized by TEM. Micrographs show localization of single tubes intra- (column A) and extracellular (column B) in healthy and COPD subjects. Inserts indicate magnified regions in columns $\mathrm{A}^{\prime}$ and $\mathrm{B}^{\prime}$ the region and the black arrows indicate the position of MWCNTs. Scale bars A,B: $1 \mu \mathrm{m}$ and A',B': $500 \mathrm{~nm}$

Figure 4: Production of pro-inflammatory cytokines and oxidative stress markers in airway epithelial cultures following exposure to MWCNTs measured by RT-qPCR. ALI cell cultures were exposed to aerosolized MWCNTs at doses of $0.16 \mu \mathrm{g} / \mathrm{cm}^{2}$ (LC, low dose) and $0.34 \mu \mathrm{g} / \mathrm{cm}^{2}\left(\mathrm{HC}\right.$, high dose). Aerosolized DQ12 $\left(0.60 \mu \mathrm{g} / \mathrm{cm}^{2}\right)$, asbestos $(\mathrm{Asb})(30 \mu \mathrm{g} / \mathrm{ml})$ and TNF- $\alpha(1 \mu \mathrm{l} / \mathrm{ml})$ as well as IFN- $\gamma(1 \mu \mathrm{l} / \mathrm{ml})$ were added as positive controls. A: RNA expression levels of pro-inflammatory cytokines IL-6, IL-8, CXCL10, IL-1 $\beta$ and TGF- $\beta$ and B: oxidative stress markers HMOX-1 and SOD-2 in cultured airway epithelial cells exposed to MWCNTs. 24h after MWCNT exposure, the total RNA content was collected. Graphs 
show single data points and mean from samples of individual donors. Statistical significance was determined by a non-parametric one-way analysis of variance (Kruskal-Wallis) followed by Dunne's post hoc test. Values were considered significantly different compared to the negative control Pluronic F127 with $\mathrm{p}<0.01(*), \mathrm{p}<0.01(* *), \mathrm{p}<0.001(* * *)$ and $\mathrm{p}<0.0001$ $(* * * *)$

Figure 5: Ciliary beating frequency before and after exposure to MWCNTs measured by high speed light microscopy. Frequency was measured in the center (A) and in periphery (B) of the culture insert. Bars show means \pm SD of 3 independent experiments.

\section{Legends SI}

Supplementary table 1: List of analyzed genes and primer sequences used for real-time PCR.

Supplementary figure 1: Production of pro-inflammatory cytokines in airway epithelial cultures following exposure to MWCNTs measured by ELISA. ALI cell cultures were exposed to aerosolized MWCNTs at doses of $0.16 \mu \mathrm{g} / \mathrm{cm}^{2}$ (LC, low dose) and $0.34 \mu \mathrm{g} / \mathrm{cm}^{2}$ (HC, high dose). Aerosolized DQ12 $\left(0.60 \mu \mathrm{g} / \mathrm{cm}^{2}\right)$, asbestos (Asb) $(30 \mu \mathrm{g} / \mathrm{ml})$ and TNF- $\alpha(1$ $\mu \mathrm{l} / \mathrm{ml})$ as well as IFN- $\gamma(1 \mu \mathrm{l} / \mathrm{ml})$ were implemented as positive controls. Cytokines secreted into the supernatant during twenty-four hours were measured using ELISA. Graphs show single data points and mean from samples of individual donors. Statistical significance was determined by a non-parametric on-way analysis of variance (Kruskal-Wallis) followed by Dunne's post hoc test. Values were considered significantly different compared to the negative control Pluronic F127 with $\mathrm{p}<0.01(*), \mathrm{p}<0.001(* * *)$ and $\mathrm{p}<0.0001(* * * *)$. 


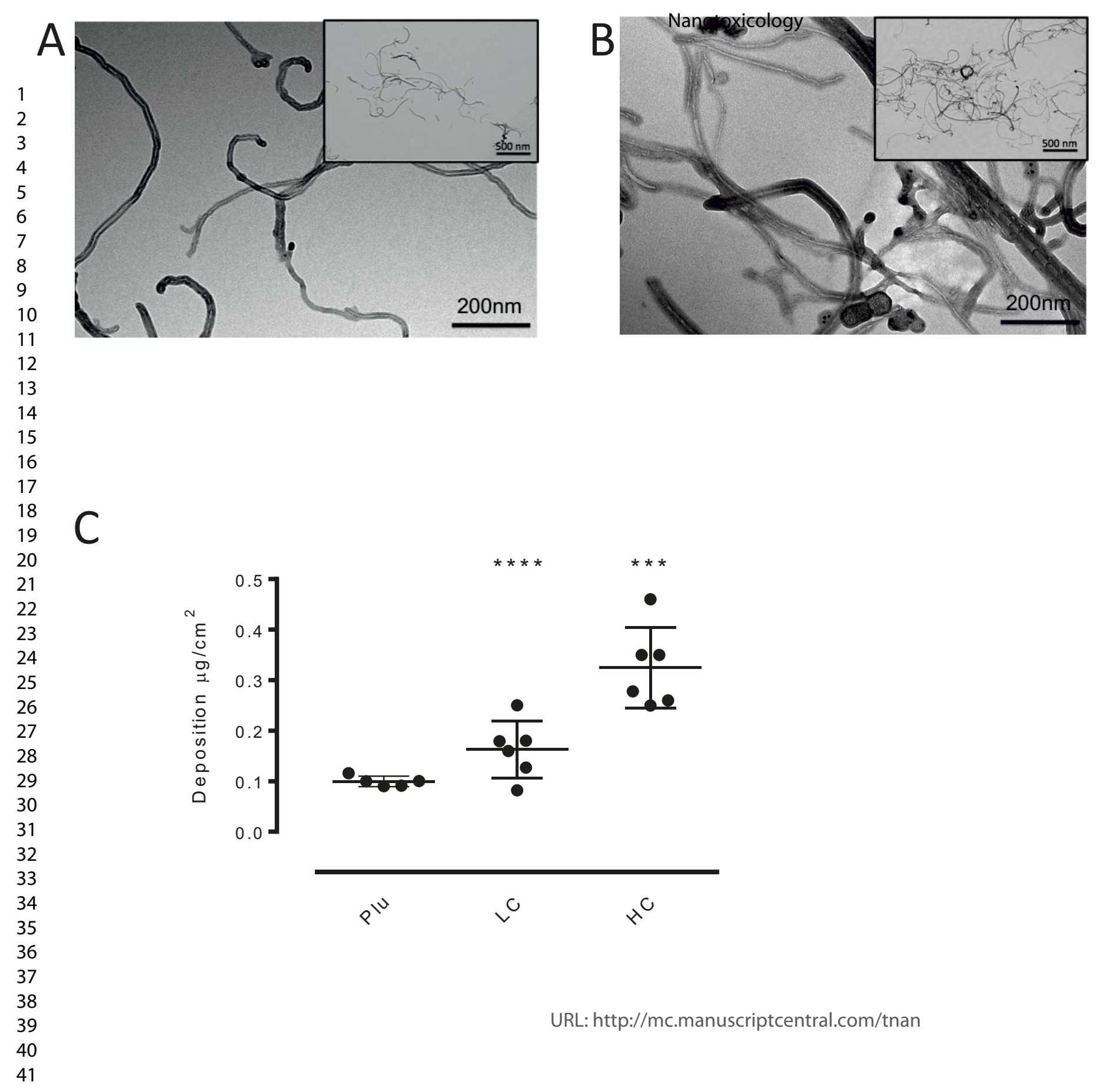

Page 32 of 45 


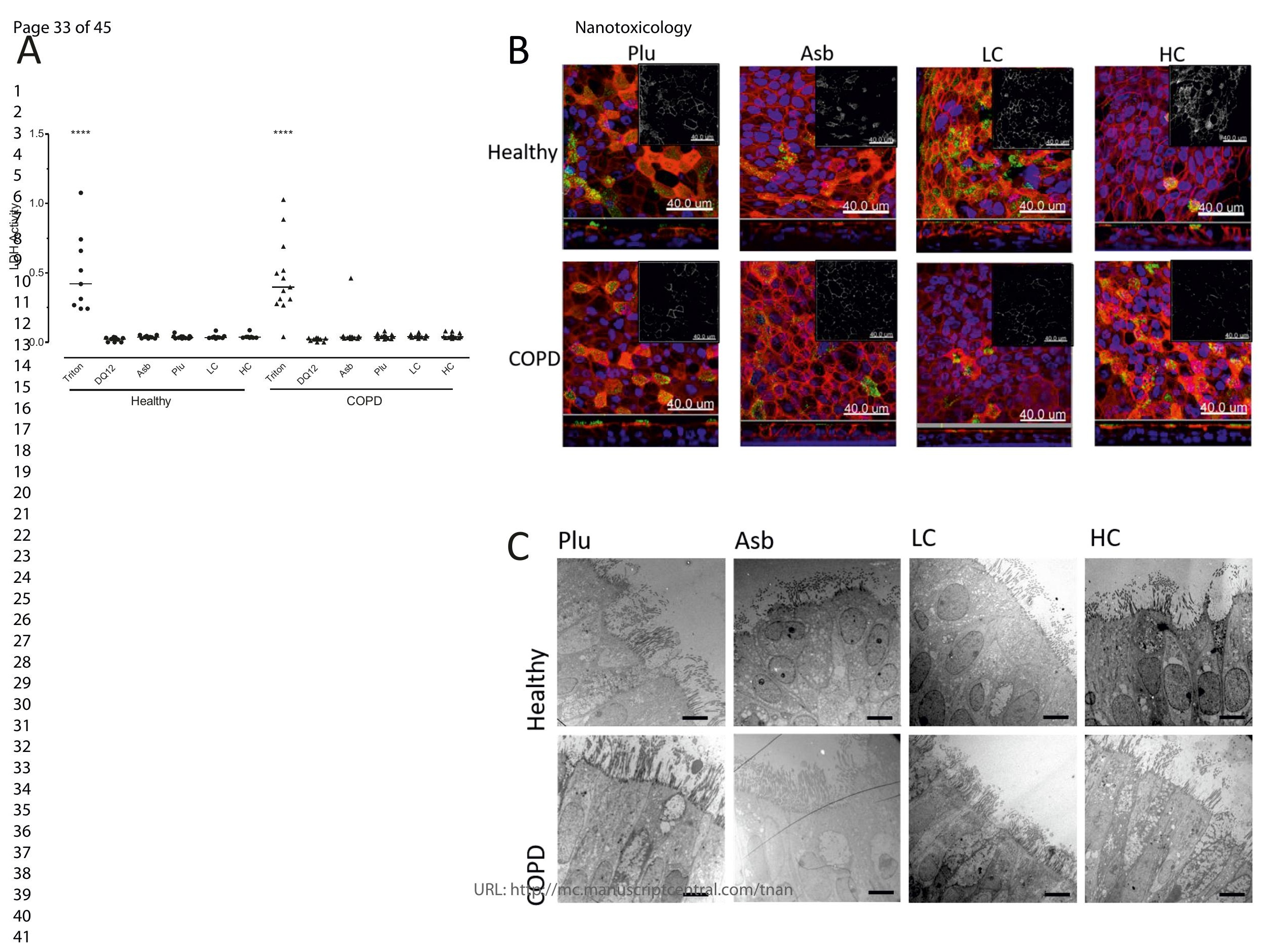




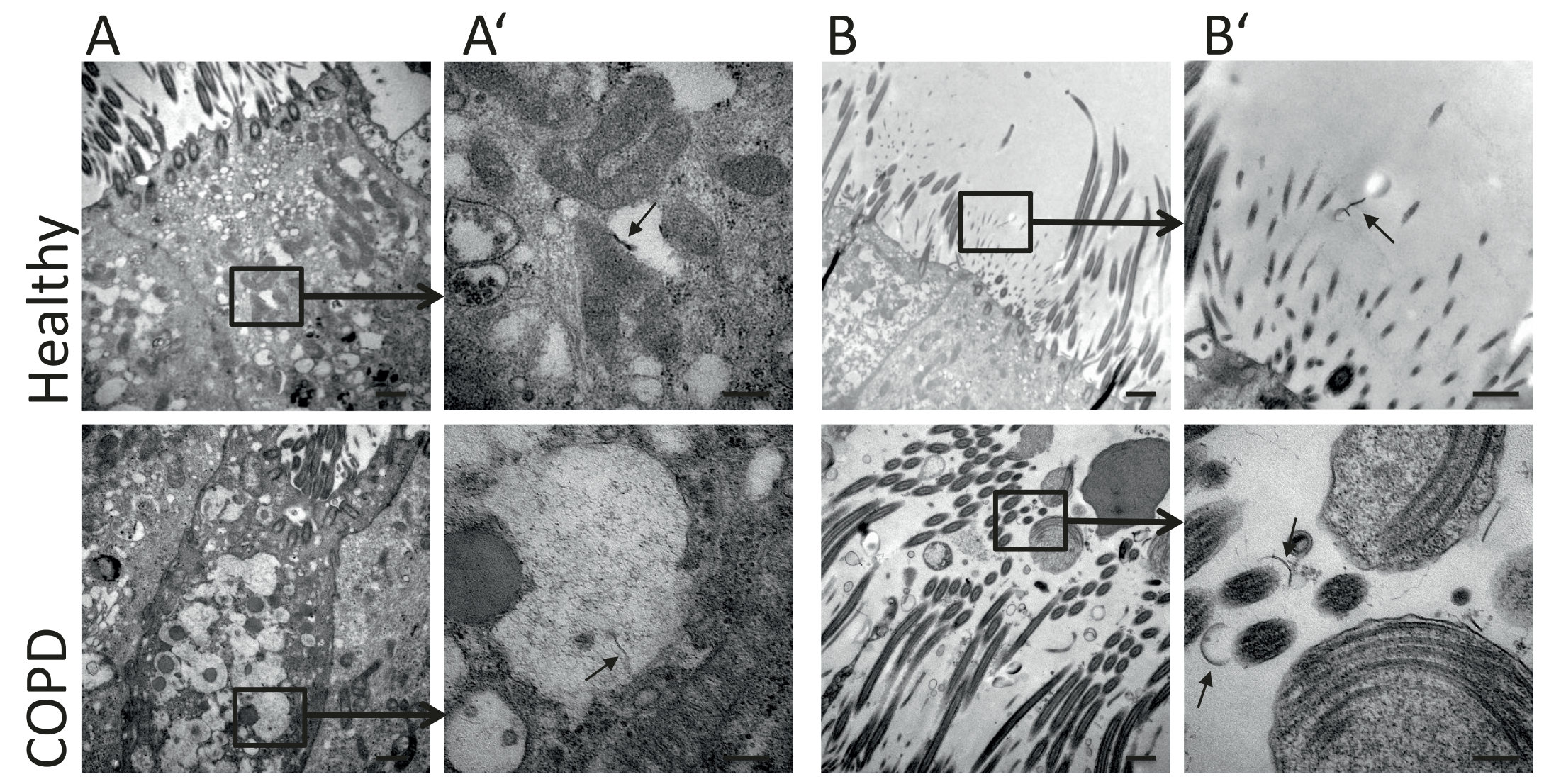



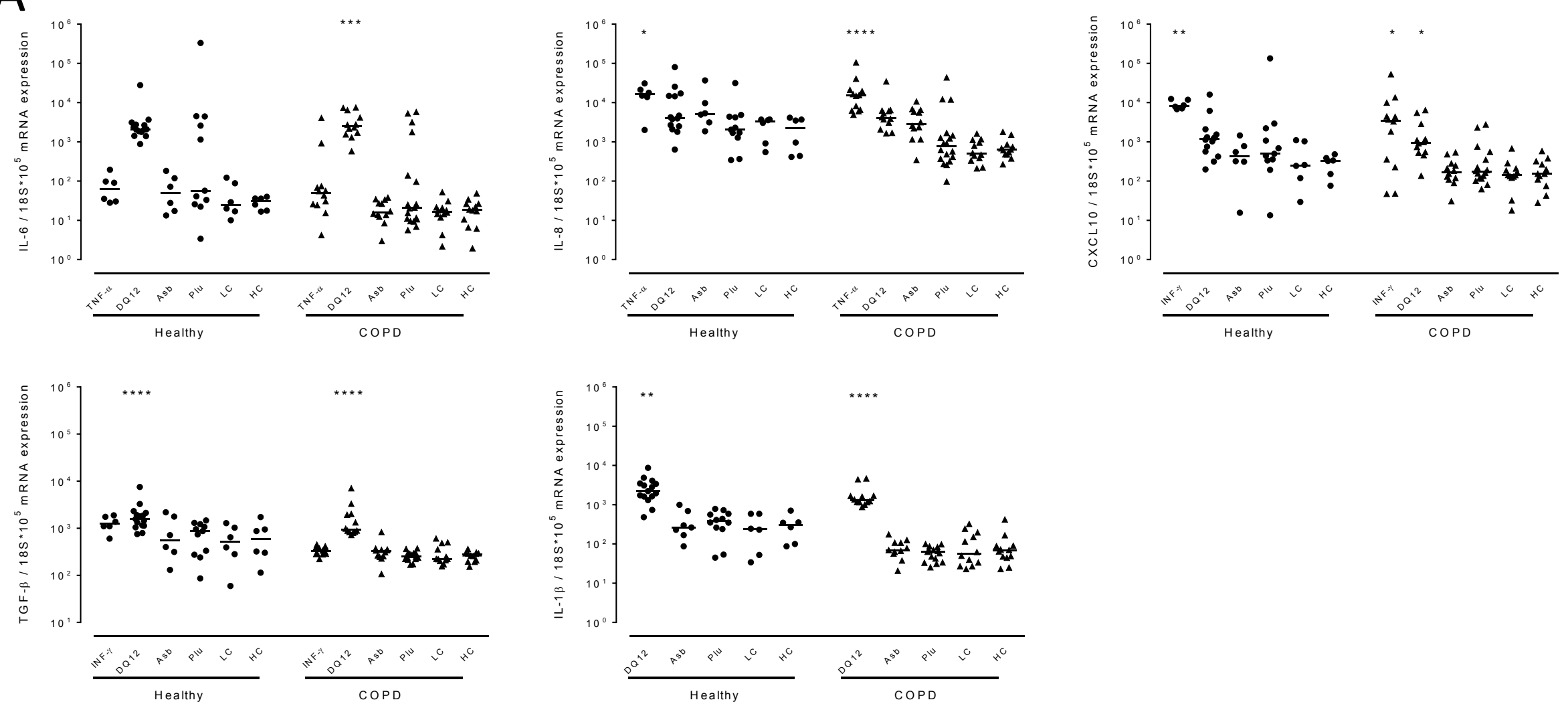

B
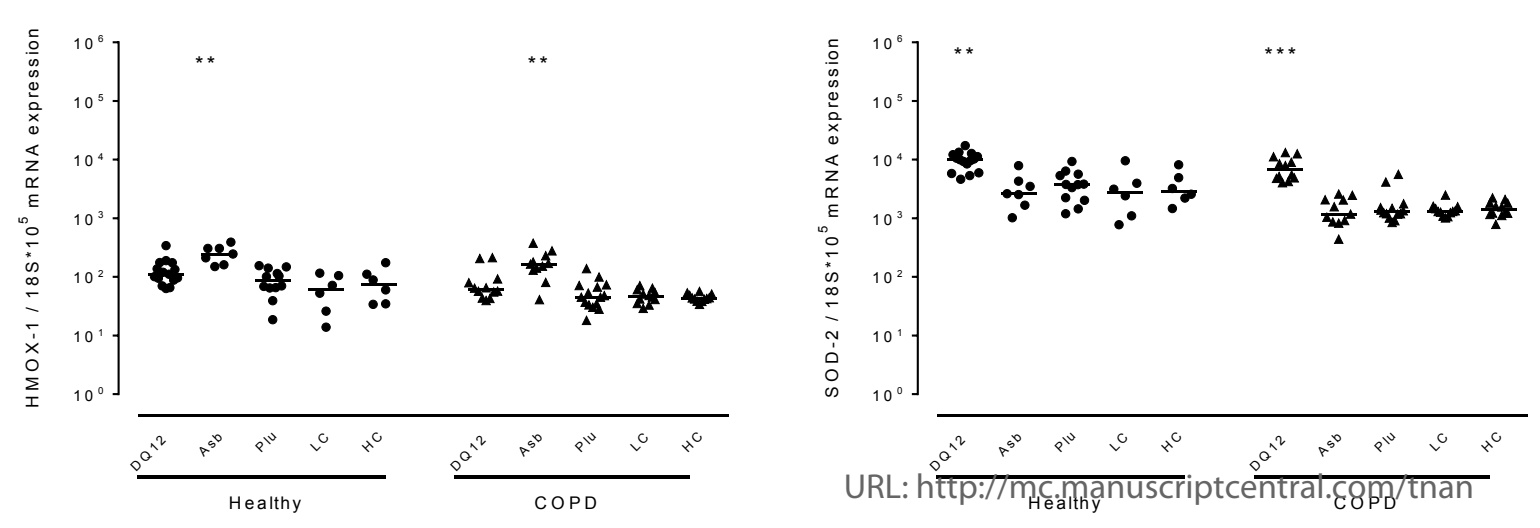
Center

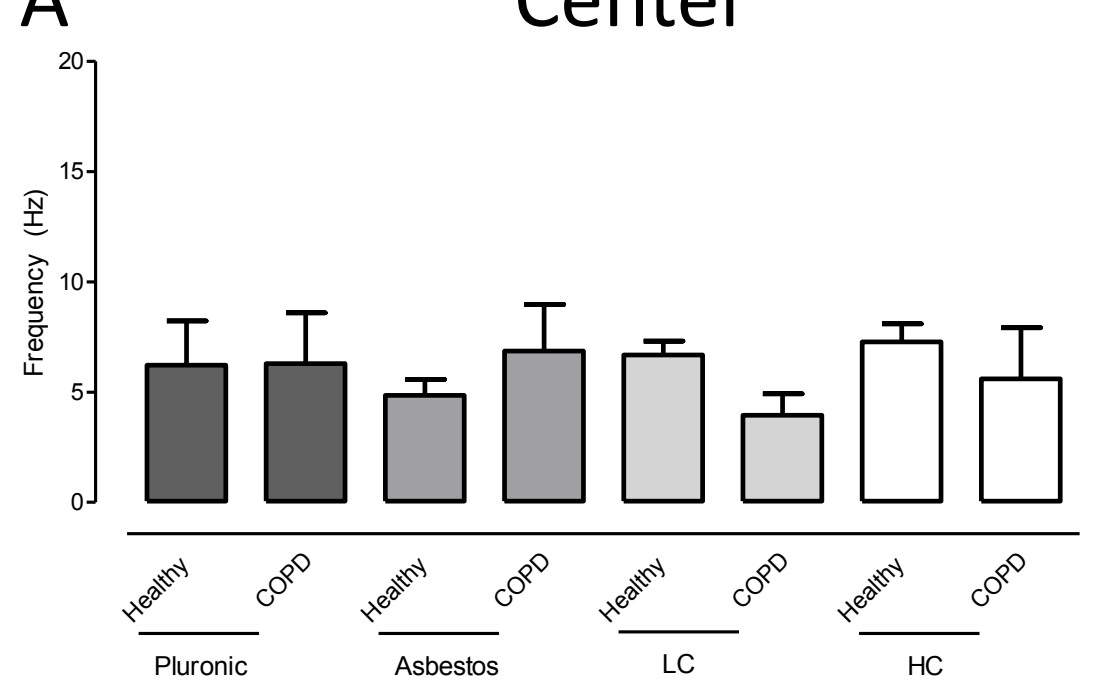

B Periphery

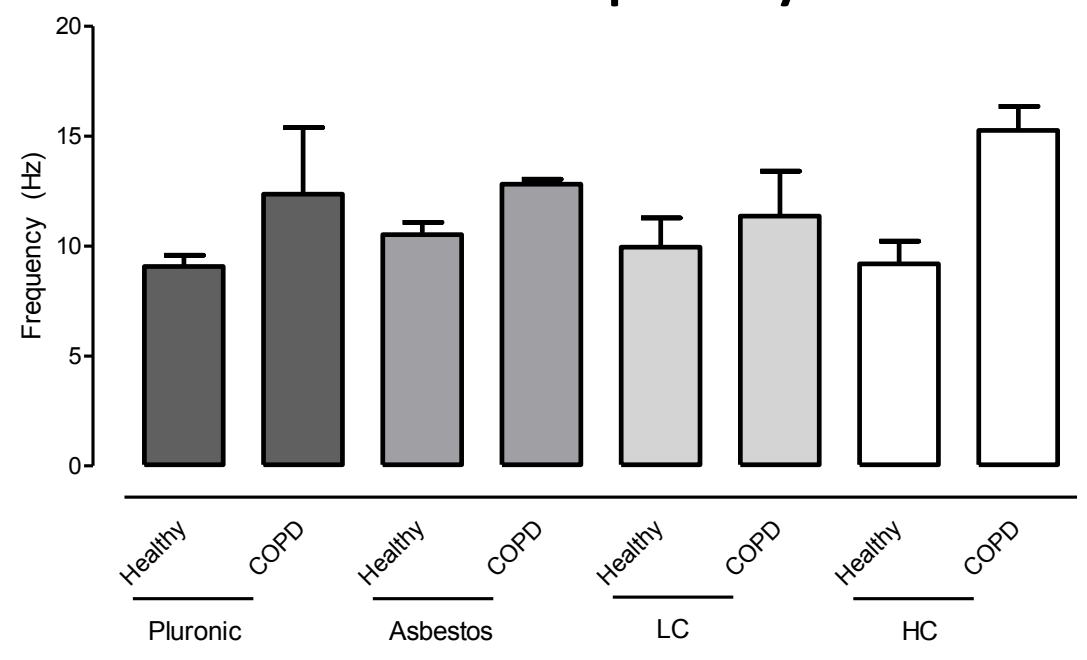



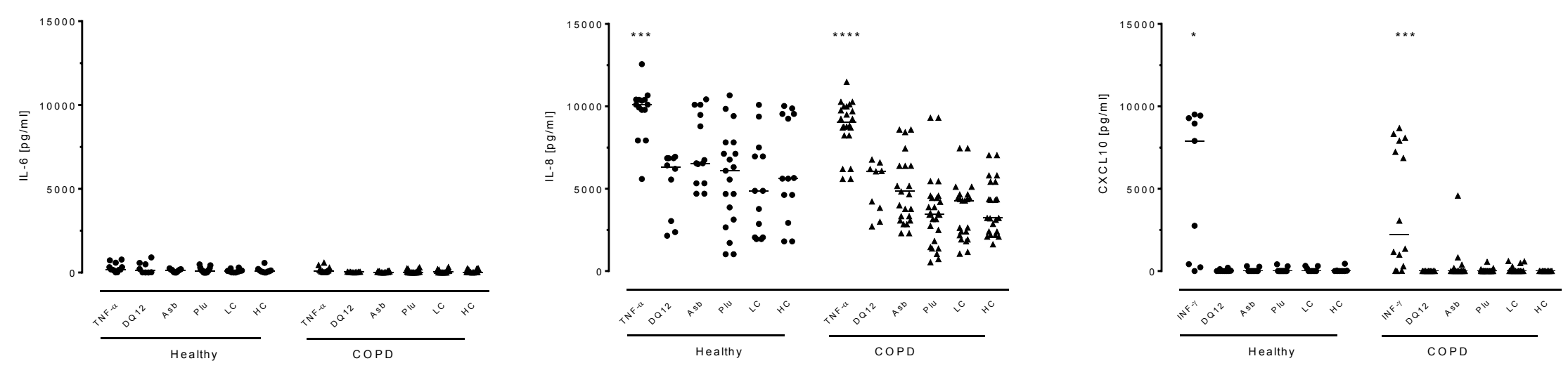
Genes

$18 S$

HMOX-1

SOD-2

IL-6

IL-8

CXCL-10

TGF- $\beta$

IL-1 $\beta$

Direction Primer Sequence

forward CGCCGCTAGAGGTGAAATTCT

reverse CATTCTTGGCAAATGCTTTCG

forward TTCTCCGATGGGTCCTTACACT

reverse GGCATAAAGCCCTACAGCAAC

forward CTGCTGGGGATTGATGTGTGG

reverse TGCAAGCCATGTATCTTTCAGT

forward CCAGGAGCCCAGCTATGAAC

reverse CCCAGGGAGAAGGCAACTG

forward CTGGCCGTGGCTCTCTTG

reverse CCTTGGCAAAACTGCACCTT

forward CCATTCTGATTTGCTGCCTTATC

reverse GCAGGTACAGCGTACAGTTCT

forward CCCTACATTTGGAGCCTGGACACG

reverse CGGGTTATGCTGGTTGTACAGGGC

forward ACGATCACTGAACTGCACGC

reverse TGTTGCTCCATATCCTGTCCC 


\section{Immunofluorescence}

Twenty-four hours after exposure to MWCNTs or asbestos, insert membranes were cut and fixed in $70 \%$ ethanol. Membranes were washed three times with washing-buffer (PBS containing $0.1 \% \mathrm{BSA}$ and $0.001 \% \mathrm{NaN}_{3}$ ). Cells were permeabilized with $0.2 \%$ Triton $\mathrm{X}-100$ for 15 minutes at room temperature. The inserts were washed three times with washingbuffer. Primary antibody mix contained rabbit anti occludin 1:100 (Invitrogen, US) and mouse anti beta tubulin 1:100 (Invitrogen, US). Antibodies were diluted in washing-buffer. Inserts were incubated for one hour at room temperature in a moisture chamber in the dark. Thereafter they were washed three times with washing-buffer. The second antibody mix contained goat anti mouse - Alexa 488 1:200 (Invitrogen, US), goat anti rabbit - Alexa 647 1:200 (Invitrogen, US) and phalloidin rhodamine 1:100 (Invitrogen, US), diluted in washingbuffer. After one hour incubation at room temperature in a moisture chamber in the dark, inserts were washed three times with washing-buffer. As much liquid, as possible was removed from the inserts, which were placed on microscopic slides and embedded in Vectashield with DAPI mounting medium (Vector Laboratories, US).

\section{Real-time qPCR}

Quantitative real-time polymerase chain reaction (RT-qPCR) was performed in a reaction volume of $20 \mu \mathrm{l}$, with a total of $2 \mu \mathrm{l}$ of fivefold diluted cDNA, by using a Fast SYBR Green master mix (Applied Biosystems, Switzerland) with $20 \mu \mathrm{M}$ forward and reverse primer (Mycrosynth, Switzerland) in a 7500 Fast Real-Time PCR System (Life Technologies, US). Settings: Denature $20 \mathrm{sec}$ at $95^{\circ} \mathrm{C}, \mathrm{PCR}$ cycles (40): $3 \mathrm{sec}$ at $95^{\circ} \mathrm{C}, 30 \mathrm{sec}$ at $60^{\circ} \mathrm{C}$. To quantify mRNA expression levels of endogenous genes, the $\Delta \Delta \mathrm{Ct}$ method was used; mRNA expression levels were normalized to $18 \mathrm{~S}$ mRNA. To examine (pro-) inflammatory responses, the expression of IL-6, IL-8, CXCL10, IL-1 $\beta$ and TGF- $\beta$ were evaluated. Oxidative stress markers HMOX-1 and SOD-2 were assessed to analyze oxidative stress. All primers were 
synthesized from Microsynth, Switzerland and the primer sequences for all tested genes can be found in SI Table 1.

\section{Transmission electron microscopy (TEM)}

Twenty-four hours after exposure to MWCNTs, asbestos or Pluronic F127, the exposed cells on membranes were cut from the inserts and fixed in buffered $2.5 \%$ glutaraldehyde (Agar Scientific Ltd., Plano GmbH, Wetzlar, Germany), containing 1.0\% osmium tetroxide (Simec, Zofingen, Switzerland). Subsequently, cells were dehydrated in a graded series of ethanol and gradual replaced by ethanol using propylene oxide prior to the infiltration and embedding of the cells into epoxy resin. Ultrathin sections were obtained utilizing the Leica Ultracut UC6 Ultramicrotome (Leica Microsystems, Vienna, Austria), before transferring on single slot uncoated copper grids. Sections were stained with uranyl acetate and counterstained with lead citrate. TEM was performed with a Fei Technai Spirit at $120 \mathrm{kV}$ (Fei Technai Spirit, Oregon, USA).

\section{Assessment of ciliary beating frequency $(\mathrm{CBF})$}

Membranes were cut and placed on a glass slide and covered with a coverslip. Microscopy was performed with an OLYMPUS Vanox-S with SPLAN Apo objectives (10x, 20x, 40x) with differential interference contrast (DIC/ Nomarski). Videos were taken randomly both in the center and periphery of the inserts using a Point Grey Flea 3 (FL3-U3-13Y3M-C) B/W high speed CMOS camera at 300 fps. Capture software was Point Grey FlyCap2 (acquisition rate $2 \mathrm{sec}$ as 320x240 Pixels at $300 \mathrm{fps}$ acquiring 600 images respectively). Motility analysis was performed in ImageJ on Java 64 bit by slow-motion image forward and manual cilia movement inspection for beat pattern and coordination analysis. For frequency analysis, the Power spectrum of ciliary oscillations was performed based on Fast Fourier Transform 
algorithm in an IDL (Interactive Data Language, Excelis / Harris Corporation, Melbourne FL, USA) module (Ryser et al. 2007) was performed. 